\title{
Supplemental Analysis to Support Postulated Events in the Process Hazards Analysis for the HEAF
}

\author{
H. E. Lambert, G. L. Johnson
}

July 20, 2001

U.S. Department of EnGroy

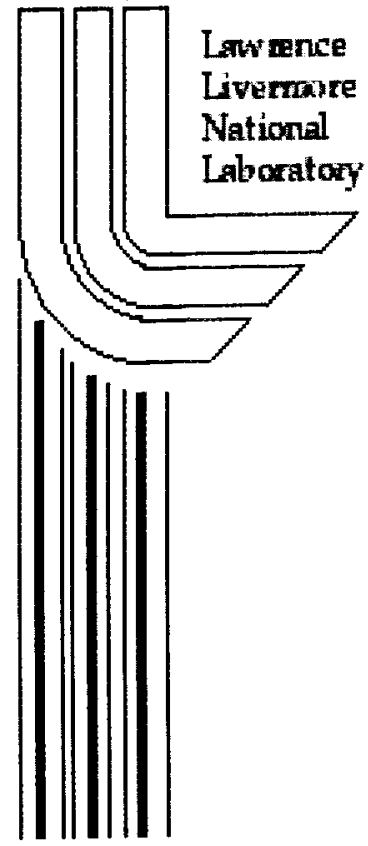




\section{DISCLAIMER}

This document was prepared as an account of work sponsored by an agency of the United States Government. Neither the United States Government nor the University of California nor any of their employees, makes any warranty, express or implied, or assumes any legal liability or responsibility for the accuracy, completeness, or usefulness of any information, apparatus, product, or process disclosed, or represents that its use would not infringe privately owned rights. Reference herein to any specific commercial product, process, or service by trade name, trademark, manufacturer, or otherwise, does not necessarily constitute or imply its endorsement, recommendation, or favoring by the United States Government or the University of California. The views and opinions of authors expressed herein do not necessarily state or reflect those of the United States Government or the University of California, and shall not be used for advertising or product endorsement purposes.

This work was performed under the auspices of the U. S. Department of Energy by the University of California, Lawrence Livermore National Laboratory under Contract No. W-7405-Eng-48.

This report has been reproduced directly from the best available copy.

Available electronically at http://www.doc.gov/bridge

Available for a processing fee to U.S. Department of Energy

And its contractors in paper from

U.S. Department of Energy

Office of Scientific and Technical Information

P.O. Box 62

Oak Ridge, TN 37831-0062

Telephone: (865) 576-8401

Facsimile: (865) 576-5728

E-mail: reports@adonis.osti.gov

Available for the sale to the public from

U.S. Department of Commerce

National Technical Information Service

5285 Port Royal Road

Springfield, VA 22161

Telephone: (800) 553-6847

Facsimile: (703) 605-6900

E-mail: orders@ntis.fedworld.gov

Online ordering: http:/ / www.ntis.gov/ordering.htm

\section{OR}

Lawrence Livermore National Laboratory

Technical Information Department's Digital Library

http://www.llnl.gov/tid/Library.html 


\subsection{Purpose of the Report}

The purpose of this report is to conduct a limit scope risk assessment by generating event trees for the accident scenarios described in table 4-2 of the HEAF SAR, ref 1. Table 4-2 lists the postulated event/scenario descriptions for non-industrial hazards for HEAF. The event tree analysis decomposes accident scenarios into basic causes that appear as branches on the event tree. Bold downward branches indicate paths leading to the accident. The basic causes include conditions, failure of administrative controls (procedural or human error events) or failure of engineered controls (hardware, software or equipment failure) that singly or in combination can cause an accident to occur. Event tree analysis is useful since it can display the minimum number of events to cause an accident. Event trees can address statistical dependency of events such as a sequence of human error events conducted by the same operator. In this case, dependent probabilities are used. Probabilities/frequencies are assigned to each branch. Another example of dependency would be when the same software is used to conduct separate actions such as activating a hard and soft crow bar for grounding detonator circuits. Generally, the first event considered in the event tree describes the annual frequency at which a specific operation is conducted and probabilities are assigned to the remaining branches. An exception may be when the first event represents a condition, then a probability is used to indicate the percentage of time the condition exists. The annual probability (frequency) of the end state leading to the accident scenario in the event tree is obtained by multiplying the branch probabilities together.

\subsection{Conduct of the study}

To conduct the study, it was important to know the hazards associated with explosives operations that include handing, processing, synthesis, transporting, storage and testing. Firing tank operations were examined in detail. A block diagram of the firing circuit (see figure 1) was generated to facilitate the understanding of how the major system components work in the shot sequence for the firing tanks. A similar scheme is used at site 300 .

\subsection{HEAF tours}

Numerous tours of HEAF were taken. The following important components were observed in the control rooms for the firing tanks:

1. System key

2. HE key

3. Green button for start

4. Red button for fire

5. Red button for stop

6. White button for reset

7. Computer displays 
The following components were examined for the run/safe system:

1. Access Entry Box

2. Run/Safe box

3. Micro Switch Door

4. Beacon

5. Door Exit box

6. Sweep box

The following components were examined for the firing tanks:

1. Tank ports

2. Ventilation system

3. Tank door and switches

4. Hydraulic system to open/close door

5. Soft crow bar (bleeder resistor)

6. Hard crow bar

7. Detonator grounding panel

and the tank diagnostic equipment that included:

1. High Speed Cameras

2. Flash Lamps

3. Laser Doppler Interferometer

4. Laser illumination and imaging

5. Heating of explosives

6. e-gun

7. X-ray heads

Numerous chemical laboratories were examined and the following were observed:

1. Fume hood ventilation system

2. Room ventilation

3. Acid Waste collection system

4. Posted weight limits and inventory

5. Storage repositories

The walls, blast doors, mazes and loading dock to HEAF were observed.

Containers used for movement of HE were observed --

1. ammunition cans

2. ice cream cartons

3. push carts

Small scale testing components were observed 
1. Drop hammer machine

2. Spark test machine

3. Friction test machine

and ODTX components that include:

1. heaters

2. holding fixtures

3. test instrumentation circuitry

4. two remote cells with shielded windows

5. shock absorbing material.

The drawers, cubicles and DOT containers were observed in magazine storage. The personal duress system was examined; specific components include

1. Transmitters

2. Receivers

3. Alarm Strobes

4. Directional Alarm Strobes

5. Alarm Horns (entrance to each room)

6. Map Display Panel

7. Annunciator Panel

8. Control Chassis \& Power Distribution.

\subsection{HEAF Personnel Discussions}

Discussions with the following individuals occurred:

William Gilliam (Facility Manager)

Roanne Lee (Lead Mechanical Engineer)

Greg Mack (Lead Electriconics Engineer)

Jim Dotts (Explosives Safety Engineer)

Denise Grimsley (1 Kg Tank Operator)

Gary Steinhour (Electronics Technician)

Ernie Urquidez (Gun Tank Operator)

Don Burns (Electronics Technican)

David Hill (Explosives Safety Engineer)

Mike Tandy (Materials Management)

Jon Maienshein (Energetic Materials Section Leader)

\subsection{Documents Reviewed}

The following documents were reviewed:

1. The ES\&H manual ref. 2 
2. The HEAF facility safety plan (FSP), ref. 3

3. Numerous HEAF control documents regarding explosives storage, operations, hazards analysis

Of particular importance to the risk assessment were two sections of the FSP -chapter 5 (entitled Hazards Analysis and Controls) and appendix C (entitled Safety Plans for Specific Operations) and the operational procedures that were referenced for these two sections.

\subsection{Risk Assessment}

Simple event trees were constructed for each hazard scenario in the HEAF SAR to help in the qualitative assessment of event occurrence probabilities. Appendix A contains these event trees. Each event in an event tree was classified into one of the probability categories shown in Table 1.

Table 1 Probability rating levels

\begin{tabular}{|l|l|l|}
\hline Category & Description & $\begin{array}{l}\text { Estimated occurrence rate } \\
\text { per year (nominal or best } \\
\text { estimate) }\end{array}$ \\
\hline Less than credible & $\begin{array}{l}\text { Events are expected not to } \\
\text { occur during the life cycle of } \\
\text { the facility. }\end{array}$ & $10^{-6}$ \\
\hline Extremely unlikely & $\begin{array}{l}\text { Events will probably not } \\
\text { occur during the life cycle of } \\
\text { the facility. }\end{array}$ & $10^{-4}$ \\
\hline Unlikely & $\begin{array}{l}\text { Events may occur once } \\
\text { during the life cycle of the } \\
\text { facility. }\end{array}$ & $10^{-2}$ \\
\hline Medium & $\begin{array}{l}\text { Event may occur during the } \\
\text { facility or operation lifetime }\end{array}$ & $10^{-1}$ \\
\hline Likely & $\begin{array}{l}\text { Events may occur several } \\
\text { times during the life cycle of } \\
\text { the facility. }\end{array}$ & 1 \\
\hline Very likely & Events may often occur. & 100 \\
\hline
\end{tabular}

The frequency at which operations are conducted was obtained from ref. 4 . The probability of each event tree branch was estimated by examining pairs of events and estimating the probability of event pairs as shown in Table 2. Where more than two events make up an event tree branch the branch probability was estimated by examining pairs of events, treating a pair as a single event and re-entering Table 2. 
The probability of a hazard scenario was taken as the probability of the most likely branch in the associated event tree. No attempt was made to sum probabilities over all branches as the trees contain relatively few branches. Summing over branches in a quantitative probability estimation scheme would affect the probability estimates by a factor of 3 to 5 . Such changes are below the resolution of the qualitative scheme adopted here.

The probability estimates of this analysis are used in the HEAF SAR summary hazard tables and together with the estimated consequences form the basis for deciding risk acceptability.

Table 2 -- Joint Probability Table (combining the probability of two events)

\begin{tabular}{|c|l|l|l|l|l|l|l|}
\hline $\begin{array}{c}\text { Annual } \\
\text { Frequency } \\
\text { Or } \\
\text { Probability }\end{array}$ & $\longrightarrow$ & $1.0 \times 10^{-6}$ & $1.0 \times 10^{-4}$ & $1.0 \times 10^{-2}$ & 0.1 & 1 & 100 \\
\hline$\downarrow$ & $\begin{array}{l}\text { Relative } \\
\text { Probability } \\
\text { Description }\end{array}$ & $\begin{array}{l}\text { Less than } \\
\text { credible }\end{array}$ & $\begin{array}{l}\text { Extremely } \\
\text { Unlikely }\end{array}$ & Unlikely & Medium & Likely & Very Unlikely \\
\hline $1.0 \times 10^{-6}$ & $\begin{array}{l}\text { Less than } \\
\text { credible }\end{array}$ & $\begin{array}{l}\text { Less than } \\
\text { credible }\end{array}$ & $\begin{array}{l}\text { Less than } \\
\text { credible }\end{array}$ & $\begin{array}{l}\text { Less than } \\
\text { credible }\end{array}$ & $\begin{array}{l}\text { Less than } \\
\text { credible }\end{array}$ & $\begin{array}{l}\text { Less than } \\
\text { credible }\end{array}$ & $\begin{array}{l}\text { Extremely } \\
\text { Unlikely }\end{array}$ \\
\hline $1.0 \times 10^{-4}$ & $\begin{array}{l}\text { Extremely } \\
\text { Unlikely }\end{array}$ & $\begin{array}{l}\text { Less than } \\
\text { credible }\end{array}$ & $\begin{array}{l}\text { Less than } \\
\text { credible }\end{array}$ & $\begin{array}{l}\text { Less than } \\
\text { credible }\end{array}$ & $\begin{array}{l}\text { Extremely } \\
\text { Unlikely }\end{array}$ & $\begin{array}{l}\text { Extremely } \\
\text { Unlikely }\end{array}$ & Unlikely \\
\hline $1.0 \times 10^{-2}$ & Unlikely & $\begin{array}{l}\text { Less than } \\
\text { credible }\end{array}$ & $\begin{array}{l}\text { Less than } \\
\text { credible }\end{array}$ & $\begin{array}{l}\text { Extremely } \\
\text { Unlikely }\end{array}$ & Unlikely & Unlikely & Likely \\
\hline 1 & Medium & $\begin{array}{l}\text { Less than } \\
\text { credible }\end{array}$ & $\begin{array}{l}\text { Extremely } \\
\text { Unlikely }\end{array}$ & Unlikely & Unlikely & Medium & Very Likely \\
\hline 100 & Likely & $\begin{array}{l}\text { Less than } \\
\text { credible }\end{array}$ & $\begin{array}{l}\text { Extremely } \\
\text { Unlikely }\end{array}$ & Unlikely & Medium & Likely & Very Likely \\
\hline
\end{tabular}

\subsection{References}

1. High Explosives Applications Facility (HEAF), Building 191, Revised July 2001, Safety Analysis Report, Lawrence Livermore National Laboratory.

2. ES\&H Manual, Lawrence Livermore National Laboratory, Part 3.1.

3. Facility Safety Plan, High Explosives Applications Facility, Building 191, FSP191, Effective September 30, 2000.

4. Memo from Carl Ingram to Howard Lambert, entitled "Estimated Activity Levels in HEAF, July 18, 2001. 


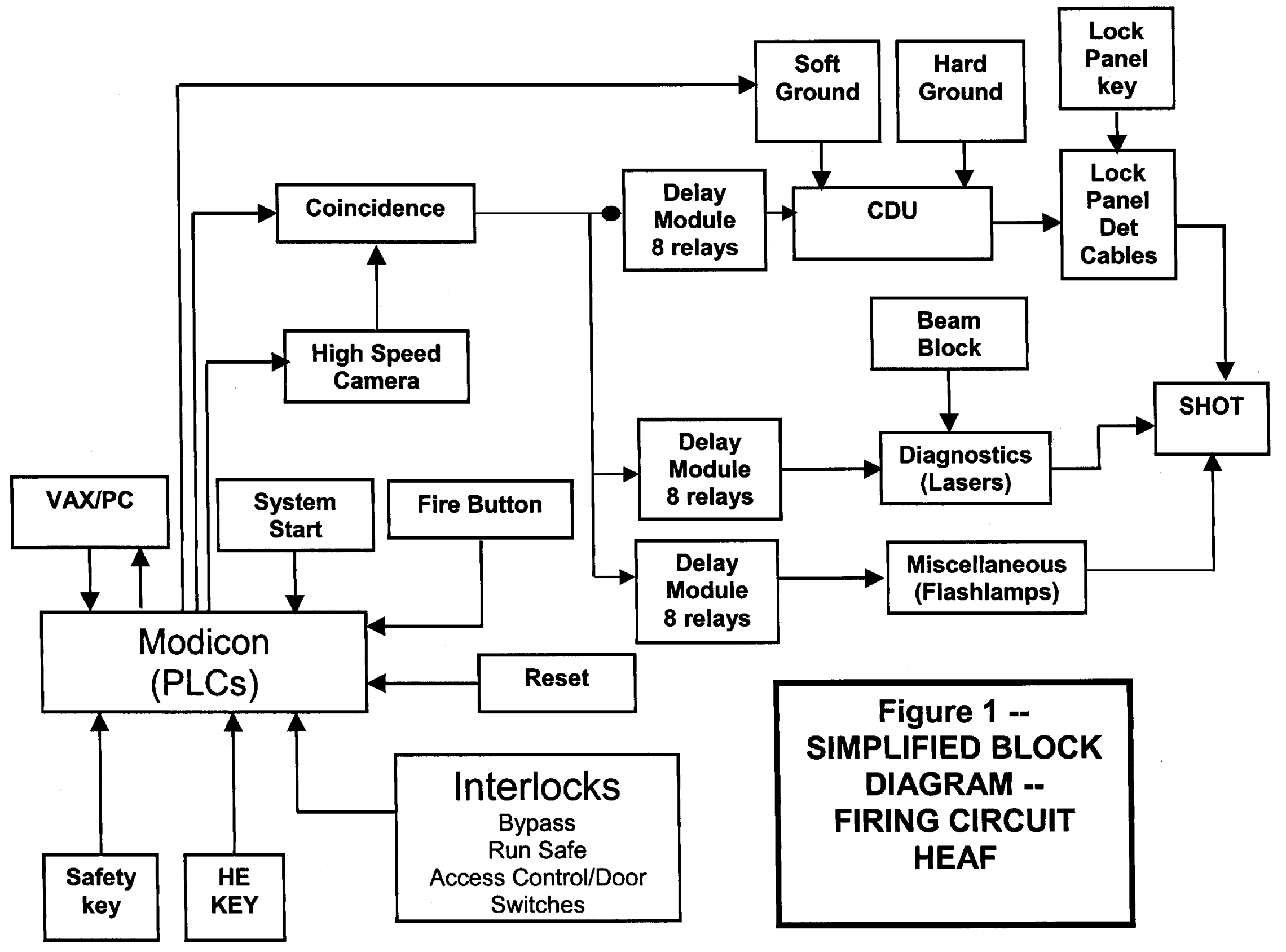




\section{APPENDIX A}

EVENT TREES 
Fig A - SAR Scenario A. Accidental Detonation or Deflagration of Explosives: Explosives are dropped or struck during handling or processing (either transport or class II operations such as assembly)

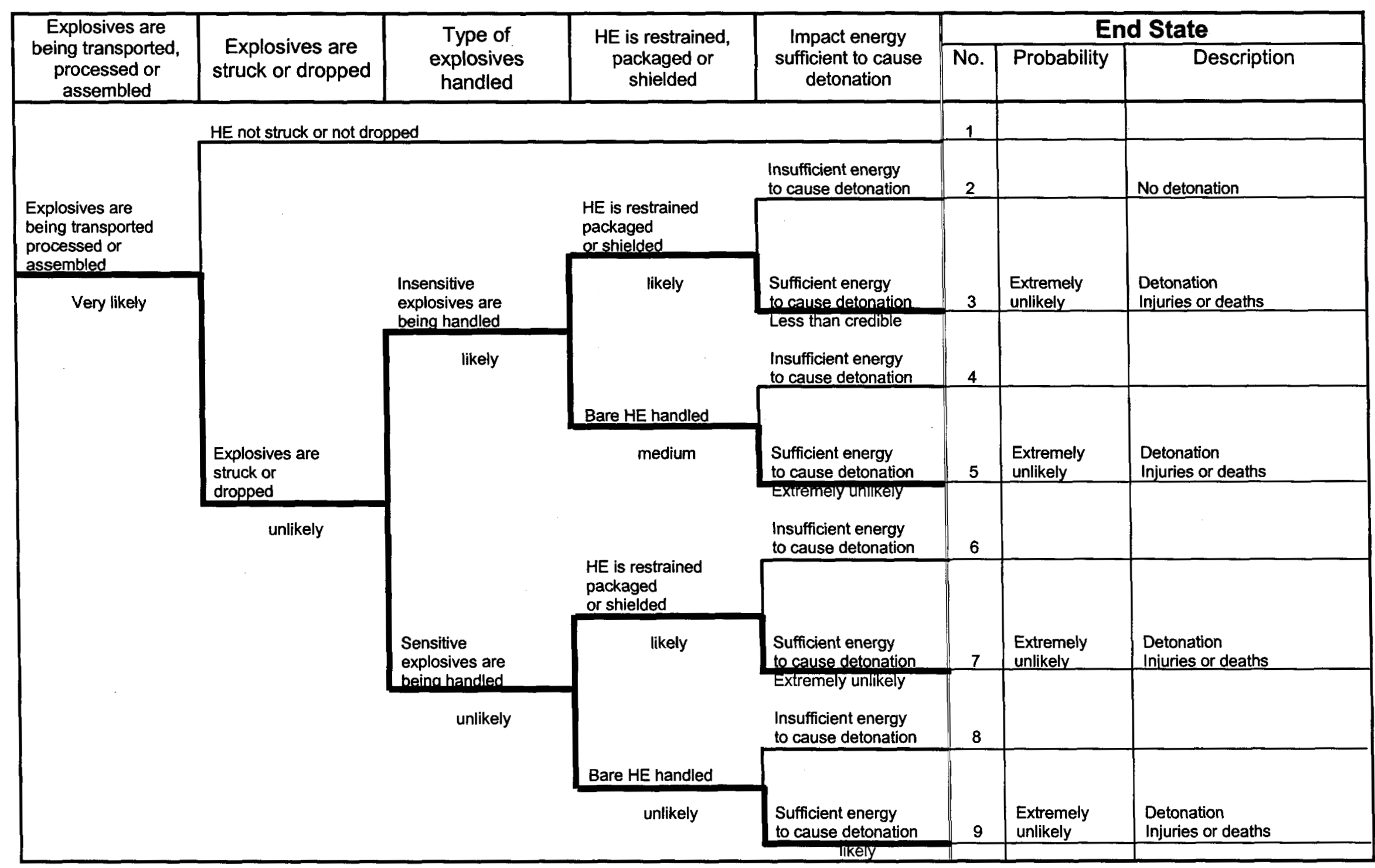


Fig B - SAR Scenario B -- Accidental Detonation or Deflagration of Explosives in storage

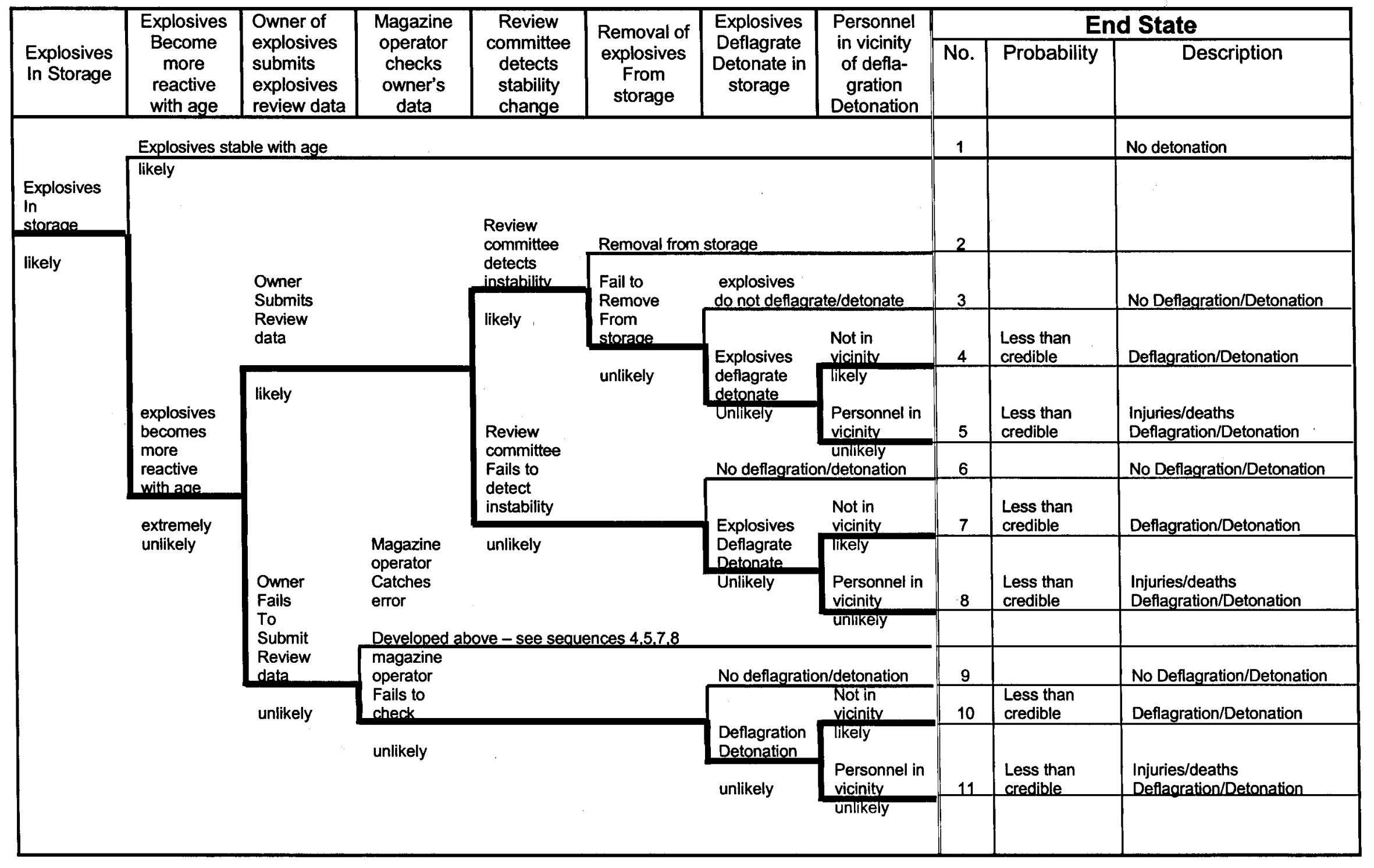


Fig C - SAR Scenario C -- Accidental Detonation Or Deflagration Of Explosives -- Intentional Detonation Experiment Prematurely Fires With Personnel In Tank Room And Tank Open

\begin{tabular}{|c|c|c|c|c|c|c|c|}
\hline \multirow[b]{2}{*}{$\begin{array}{c}\text { Personnel are } \\
\text { setting up } \\
\text { experiment with HE } \\
\text { in firing tank }\end{array}$} & \multicolumn{3}{|c|}{ Grounding } & \multirow[b]{2}{*}{$\begin{array}{l}\text { CDU Charged and } \\
\text { Fired while detonators } \\
\text { cables are being } \\
\text { hooked up to HE }\end{array}$} & \multicolumn{3}{|c|}{ End State } \\
\hline & $\begin{array}{l}\text { Detonator Cables } \\
\text { (Administrative } \\
\text { Control) }\end{array}$ & $\begin{array}{l}\text { Hard Crow Bar } \\
\text { (Administrative } \\
\text { Control) }\end{array}$ & $\begin{array}{l}\text { Soft Crow Bar } \\
\text { Bleeder Resistor } \\
\text { (Administrative } \\
\text { Control) } \\
\end{array}$ & & No. & $\begin{array}{c}\text { Annual } \\
\text { Probability }\end{array}$ & Description \\
\hline \multirow{2}{*}{$\begin{array}{l}\text { Personnel are setting } \\
\text { up experiment in } \\
\text { firing tank }\end{array}$} & Detonators grounded & & & & 1 & & NO DETONATION \\
\hline & & \multicolumn{3}{|c|}{ Hard crow bar installed and working properly } & 2 & & NO DETONATION \\
\hline \multirow[t]{5}{*}{ Very likely } & $\begin{array}{l}\text { Detonators } \\
\text { not grounded -- } \\
\text { administrative error }\end{array}$ & \multirow[b]{2}{*}{$\begin{array}{l}\text { Hard crow bar } \\
\text { not installed - } \\
\text { administrative error }\end{array}$} & & & 3 & & NO DETONATION \\
\hline & unlikely & & \multirow{2}{*}{$\begin{array}{l}\text { Soft crow bar } \\
\text { not installed -- } \\
\text { Administrative error }\end{array}$} & \multirow{2}{*}{$\frac{\text { CDU not charged }}{\text { likely }}$} & 4 & & NO DETONATION \\
\hline & & $\begin{array}{c}\text { medium } \\
\text { (dependent probability) }\end{array}$ & & & & & \\
\hline & & & \multirow{2}{*}{$\begin{array}{c}\text { likely } \\
\text { (dependent probability) }\end{array}$} & $\begin{array}{l}\text { CDU charged and } \\
\text { fired - software failure }\end{array}$ & 5 & $\begin{array}{l}\text { extremely } \\
\text { unlikely }\end{array}$ & $\begin{array}{l}\text { DETONATION - MULTIPLE } \\
\text { INJURIES OR DEATH }\end{array}$ \\
\hline & & & & extremely unlikely & & & \\
\hline
\end{tabular}


D. Accidental detonation or deflagration of explosives: explosives are initiated by electrical energy from adjacent equipment or utilities

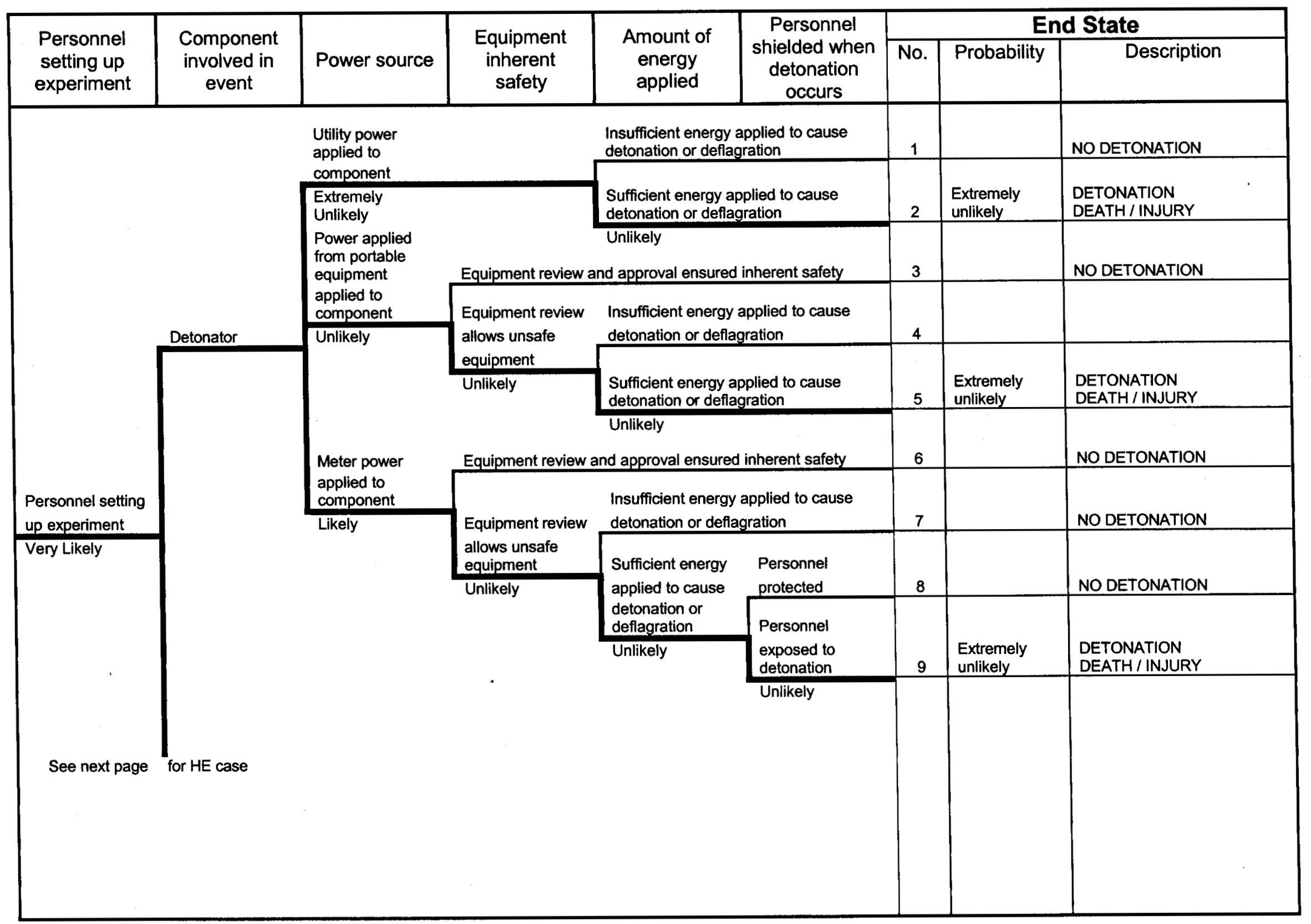







Fig E-SAR Scenario E Accidental Detonation or Deflagration of Explosives: Explosives are initiated by electrical energy from electronic gun

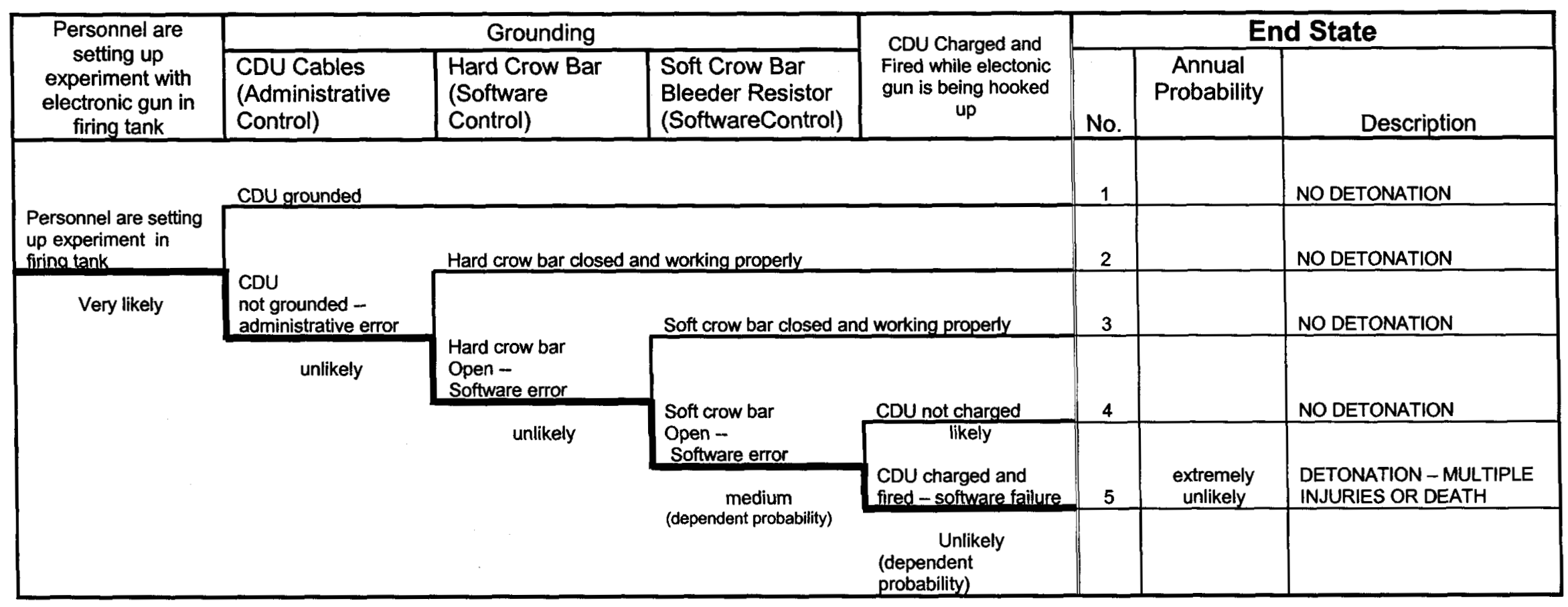


Fig F - SAR Scenario F. Accidental Detonation or Deflagration of Explosives: Explosives are initiated by nearby fire

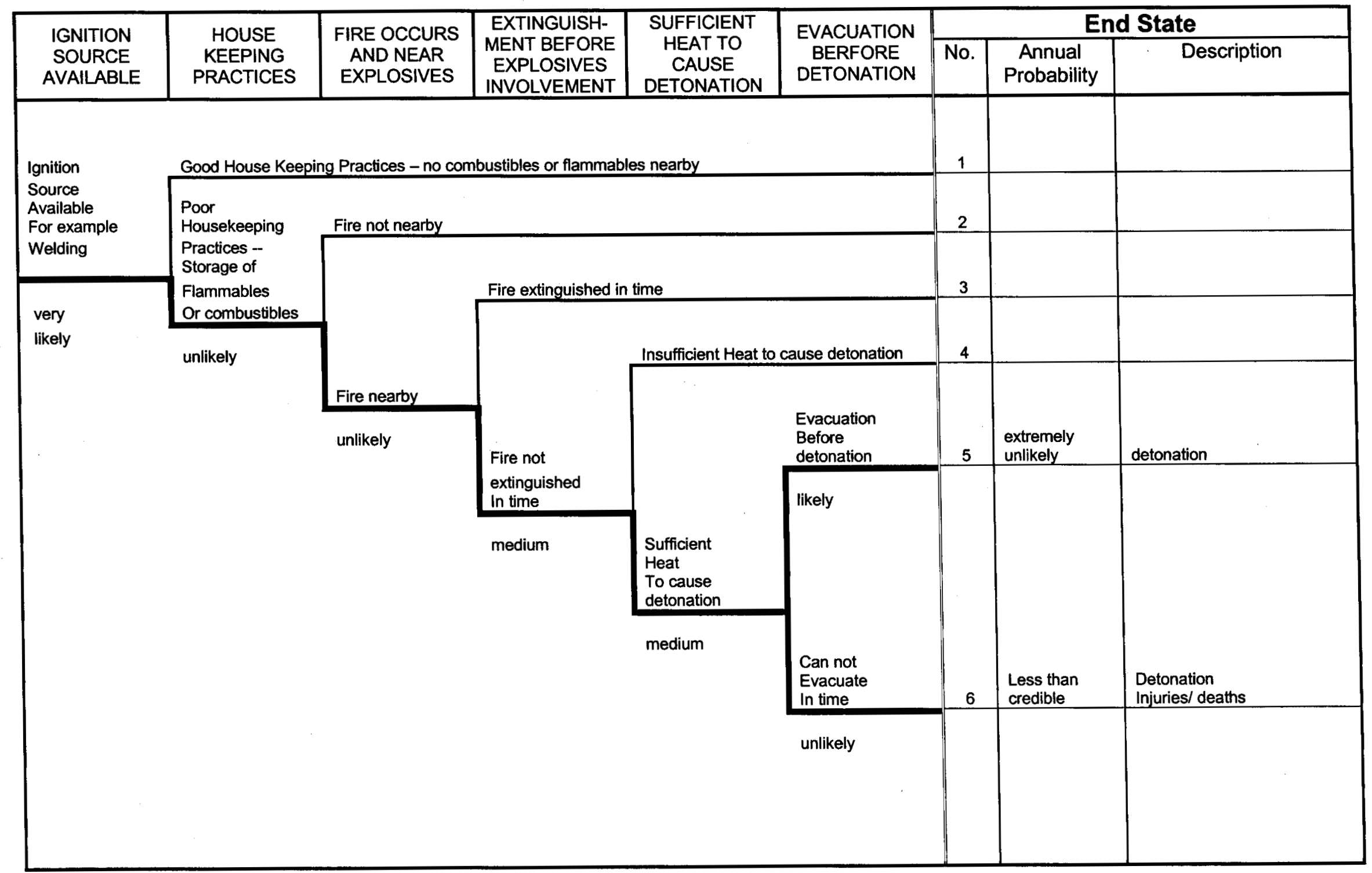


Fig G. SAR Scenario G Accidental detonation or deflagration of explosives: explosives are initiated by chemical reaction occurring nearby

\begin{tabular}{|c|c|c|c|c|c|c|c|c|}
\hline \multirow[b]{2}{*}{$\begin{array}{c}\text { Chemical } \\
\text { processing } \\
\text { near HE }\end{array}$} & \multirow[b]{2}{*}{$\begin{array}{l}\text { Chemical } \\
\text { compatibility }\end{array}$} & \multirow{2}{*}{$\begin{array}{c}\text { Peer review } \\
\text { detects } \\
\text { compatibility } \\
\text { issue }\end{array}$} & \multirow[b]{2}{*}{$\begin{array}{l}\text { Incompatible } \\
\text { chemicals } \\
\text { violently react }\end{array}$} & \multirow{2}{*}{$\begin{array}{c}\text { Reaction of } \\
\text { incompatible } \\
\text { chemicals } \\
\text { detonates HE }\end{array}$} & \multirow{2}{*}{$\begin{array}{c}\text { Personnel } \\
\text { shielded when } \\
\text { detonation } \\
\text { occurs }\end{array}$} & \multicolumn{3}{|c|}{ End State } \\
\hline & & & & & & No. & Probability & Description \\
\hline \multirow{2}{*}{$\begin{array}{l}\text { Chemical } \\
\text { processing near } \\
\text { HE }\end{array}$} & \multicolumn{5}{|c|}{ Chemicals are compatible or compatibility issues are adequately addressed } & 1 & & NO DETONATION \\
\hline & \multirow{2}{*}{$\begin{array}{l}\text { Hazards of } \\
\text { incompatible } \\
\text { chemicals not } \\
\text { adequately } \\
\text { addressed }\end{array}$} & \multicolumn{4}{|c|}{ Compatlbility issues are adequately addressed } & 2 & & NO DETONATION \\
\hline \multirow[t]{7}{*}{ Very likely } & & \multirow{2}{*}{$\begin{array}{l}\text { Peer } \\
\text { Review } \\
\text { Fails to } \\
\text { Detect } \\
\text { incompatibility }\end{array}$} & \multicolumn{3}{|c|}{ Violent reaction does not occur } & 3 & & NO DETONATION \\
\hline & Unlikely & & \multicolumn{3}{|c|}{$\begin{array}{l}\text { Reactlon does not cause HE } \\
\text { detonation or deflagration }\end{array}$} & 4 & $\begin{array}{l}\text { Extremely } \\
\text { Unlikely }\end{array}$ & $\begin{array}{l}\text { VIOLENT CHEMICAL } \\
\text { REACTION } \\
\text { INJURY } \\
\end{array}$ \\
\hline & & Unlikely & $\begin{array}{l}\text { Violent chemical } \\
\text { reaction }\end{array}$ & \multirow[b]{3}{*}{$\begin{array}{l}\text { Reaction causes } \\
\text { HE detonation or } \\
\text { deflagration }\end{array}$} & \multirow{2}{*}{$\begin{array}{l}\text { Personnel not } \\
\text { exposed } \\
\text { To } \\
\text { detonation }\end{array}$} & & \multirow{2}{*}{$\begin{array}{l}\text { Less } \\
\text { Than } \\
\text { Credible }\end{array}$} & \multirow[b]{2}{*}{ DETONATION } \\
\hline & & & Unlikely & & & 5 & & \\
\hline & & & $\begin{array}{l}\text { To } \\
\text { Extremely } \\
\text { unlikely }\end{array}$ & & \multirow{2}{*}{$\begin{array}{l}\text { Likely } \\
\text { Personnel } \\
\text { exposed to } \\
\text { detonation }\end{array}$} & & \multirow[b]{2}{*}{$\begin{array}{l}\text { Less } \\
\text { Than } \\
\text { Credible }\end{array}$} & \multirow[b]{2}{*}{$\begin{array}{l}\text { DETONATION } \\
\text { INJURY I DEATH } \\
\end{array}$} \\
\hline & & & & \multirow[t]{2}{*}{ Unlikely } & & 6 & & \\
\hline & & & & & medium & & & \\
\hline
\end{tabular}


Fig. H. - SAR SCENARIO H -- Accidental detonation or deflagration of explosives: explosives are initiated or sensitized and initiated by normal handling by reaction from chemical incompatibility

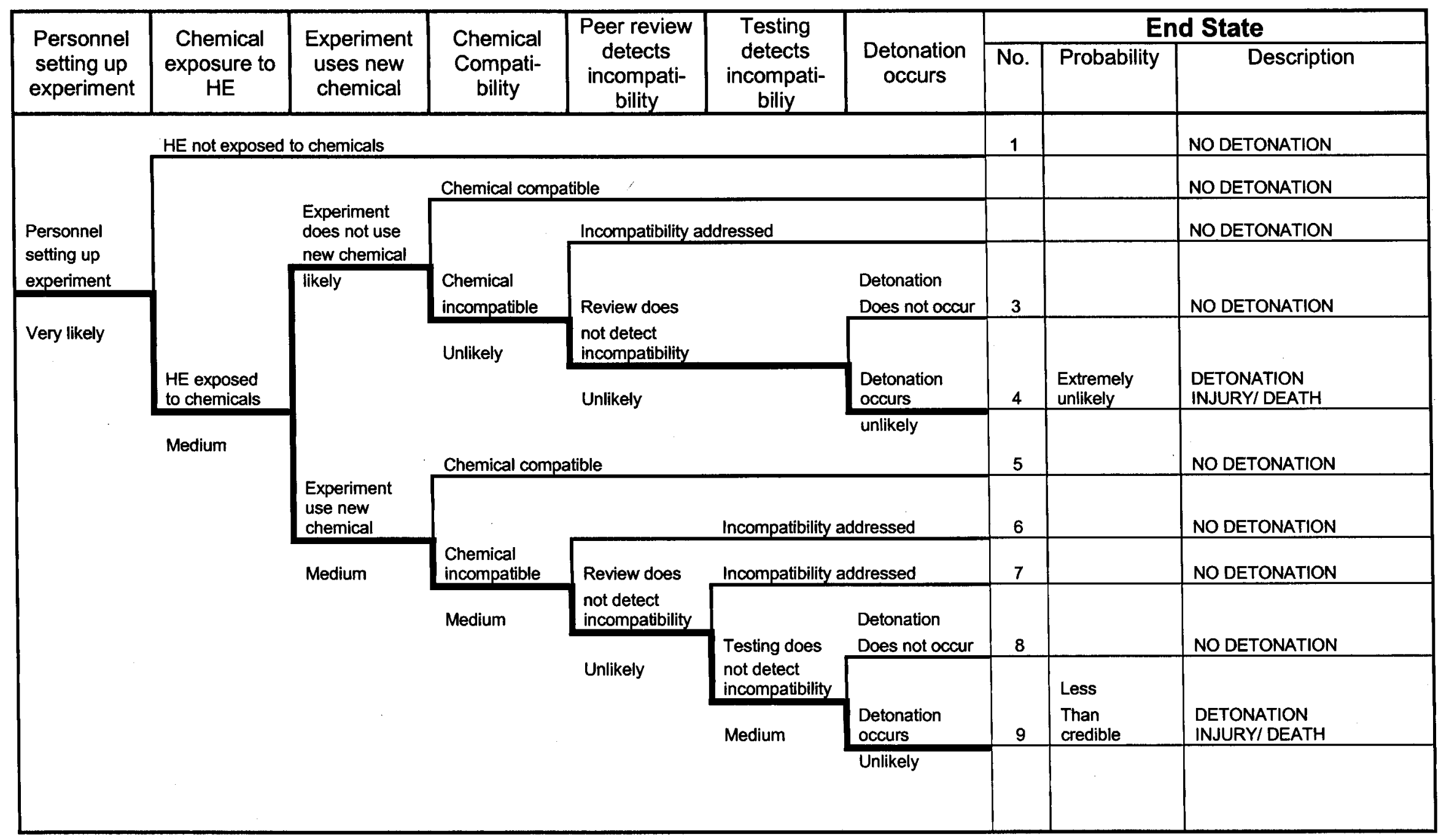


Fig I-SAR Scenario I -- Accidental Detonation or Deflagration of Explosives: Explosives are initiated by heating from laser

\begin{tabular}{|c|c|c|c|c|c|c|}
\hline \multirow{2}{*}{$\begin{array}{c}\text { OPERATIONS INVOLVING } \\
\text { USE OF HIGH POWERED } \\
\text { LASER }\end{array}$} & \multirow{2}{*}{$\begin{array}{c}\text { OPERATIONS INVOLVING } \\
\text { USE OF HIGH POWERED } \\
\text { LASER }\end{array}$} & \multirow{2}{*}{$\begin{array}{l}\text { Laser shutter } \\
\text { position }\end{array}$} & \multirow{2}{*}{$\begin{array}{l}\text { HE DETONATES DUE TO } \\
\text { OVERTEMPERATUE }\end{array}$} & \multicolumn{3}{|c|}{ End State } \\
\hline & & & & No. & Probability & Description \\
\hline \multirow[b]{3}{*}{$\begin{array}{l}\text { Operations involving the use } \\
\text { of high powered lasers }\end{array}$} & Control Circuit Works & & & 1 & & No detonation \\
\hline & \multirow[b]{3}{*}{$\begin{array}{l}\text { Power control circuit } \\
\text { For laser fails full on } \\
\text { Software failure }\end{array}$} & \multicolumn{2}{|l|}{ Laser Shutter remains closed } & 2 & & No detonation \\
\hline & & \multirow[b]{3}{*}{$\begin{array}{l}\text { Laser shutter inadvertently } \\
\text { opens - } \\
\text { Independent software failure }\end{array}$} & \multirow[b]{2}{*}{ HE does not detonate } & \multirow[b]{2}{*}{3} & & \multirow[b]{2}{*}{ No detonation } \\
\hline \multirow[t]{4}{*}{ Very likely } & & & & & & \\
\hline & $\begin{array}{l}\text { Extremely } \\
\text { unlikely }\end{array}$ & & & \multirow[b]{2}{*}{4} & \multirow[b]{2}{*}{$\begin{array}{l}\text { Less } \\
\text { Than } \\
\text { credible }\end{array}$} & \multirow[b]{2}{*}{ Detonation/Injuries or Deaths } \\
\hline & & \multirow[t]{2}{*}{$\begin{array}{l}\text { Extremely } \\
\text { Unlikely }\end{array}$} & $\begin{array}{l}\text { HE overheats } \\
\text { And detonates }\end{array}$ & & & \\
\hline & & & unlikely & & & \\
\hline
\end{tabular}


Fig J. SAR scenario J-- Accidental detonation or deflagration of explosives: Explosivers are initiated from normal stimuli after synthesis of unusually sensitive intermediate (or final) product.

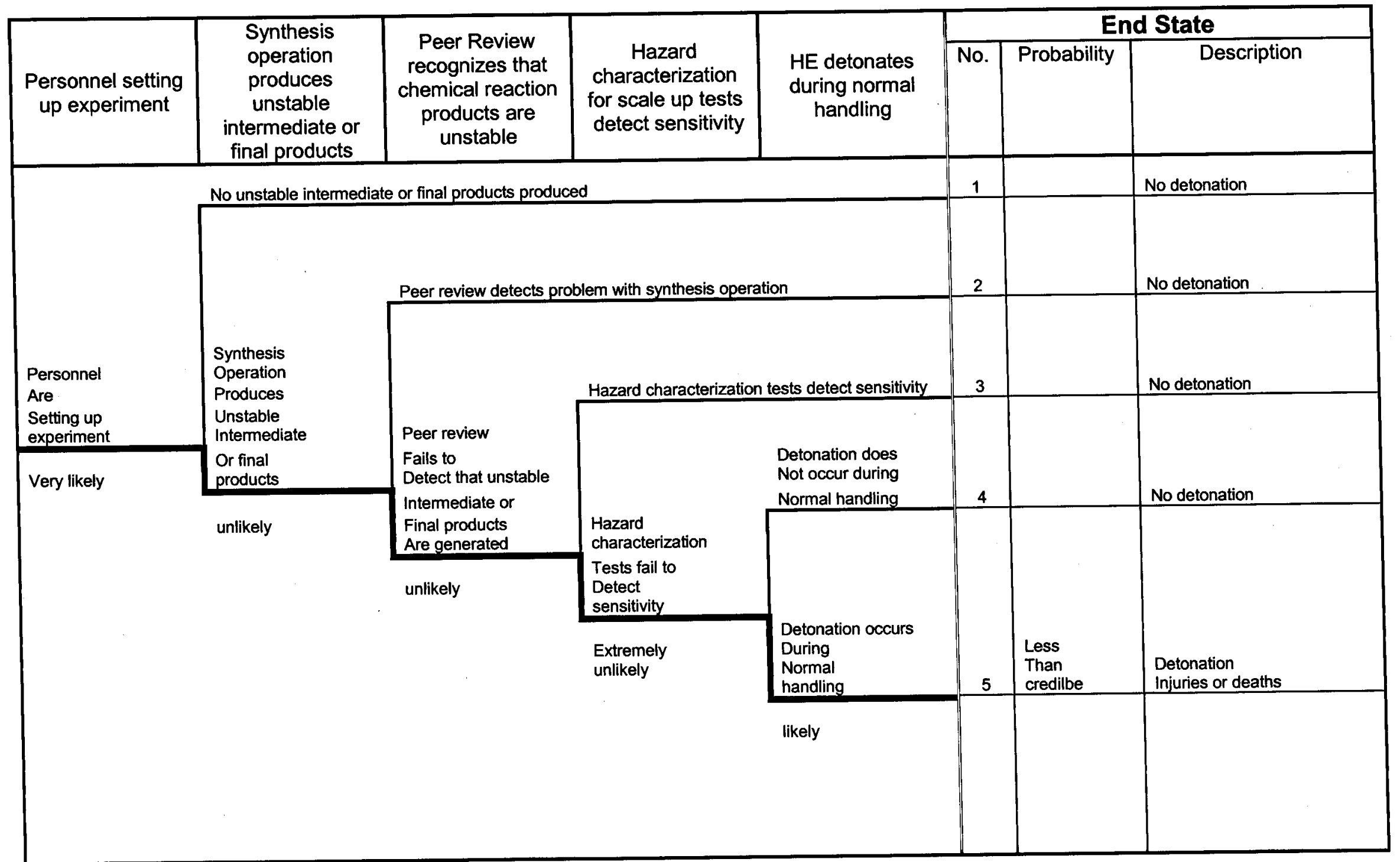


Fig K - SAR Scenario K. Accidental Detonation or Deflagration of Explosives: Explosives are initiated by unintentional heating to critical temperature.

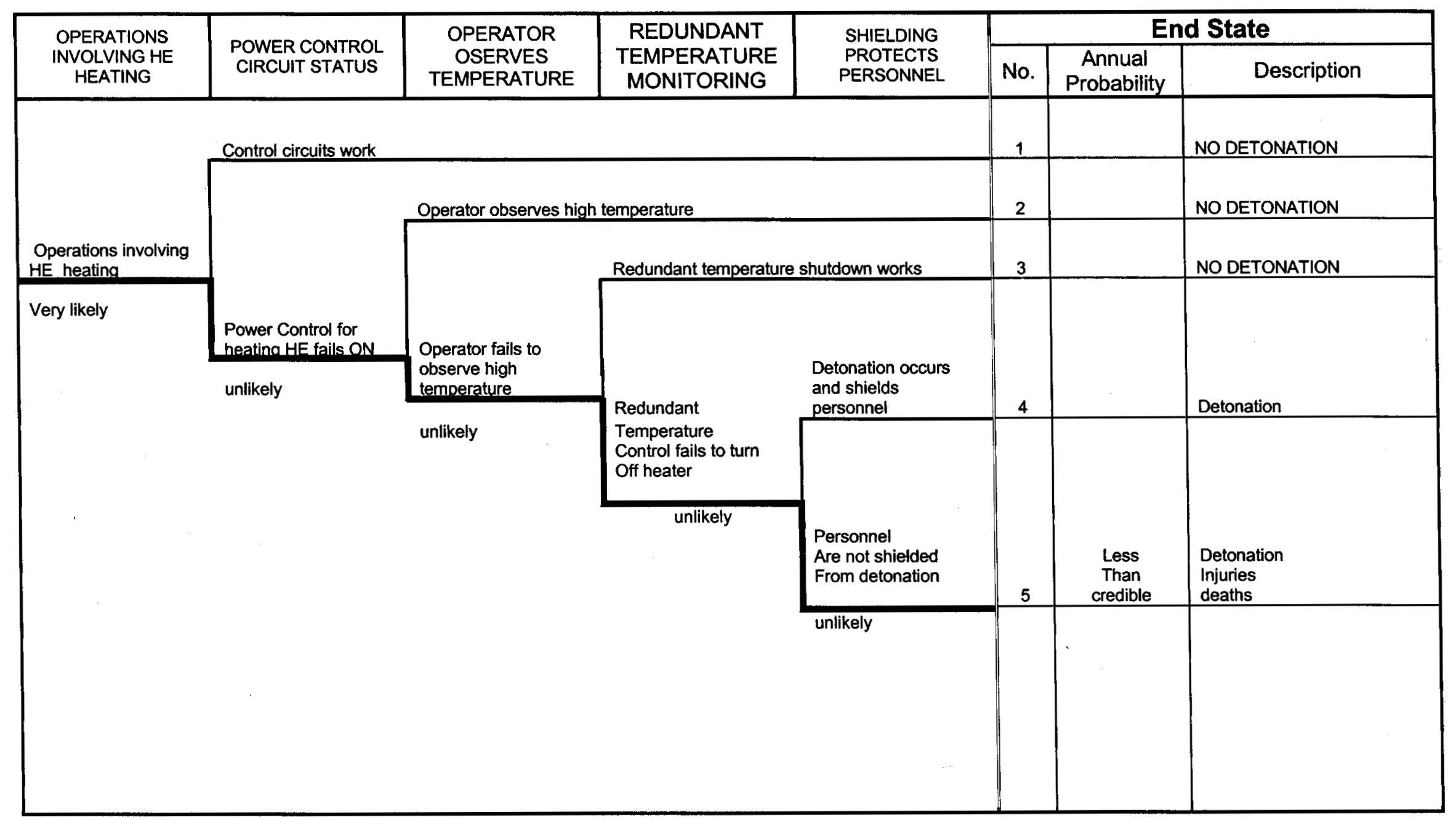


Fig L. SAR Scenario L -- Intentional detonation: Firing tank is breached or $100 \mathrm{~mm}$ gun breach fails




Fig. M SAR Scenario M -- Chemical dispersion/release

\begin{tabular}{|c|c|c|c|c|c|}
\hline \multirow[b]{2}{*}{$\begin{array}{c}\text { OPERATIONS ARE } \\
\text { OCCURRING IN CHEMICAL } \\
\text { LABORATIES }\end{array}$} & \multirow{2}{*}{$\begin{array}{c}\text { INIATING EVENT THAT } \\
\text { COULD CAUSE HAZARDOUS } \\
\text { CHEMICAL RELEASE IN } \\
\text { CHEMICAL LABORATORIES }\end{array}$} & \multirow[b]{2}{*}{$\begin{array}{l}\text { HOODNENTILATION } \\
\text { SYSTEM MITIGATES } \\
\text { CHEMICAL RELEASE }\end{array}$} & \multirow[b]{2}{*}{ No. } & \multirow[b]{2}{*}{$\begin{array}{l}\text { AnnualP } \\
\text { Probability }\end{array}$} & \multirow[b]{2}{*}{ Description } \\
\hline & & & & & \\
\hline \multirow{7}{*}{$\begin{array}{l}\text { Operations } \\
\text { Are occurring } \\
\text { In } \\
\text { Chemical } \\
\text { laboratories }\end{array}$} & Exothermic Chemical Reaction & $\begin{array}{l}\text { Hood ventilation } \\
\text { System mitigates } \\
\text { Chemical release } \\
\end{array}$ & 1 & & \\
\hline & Due to human error & \multirow{2}{*}{$\begin{array}{l}\text { Hood ventilation } \\
\text { System fails to mitigate } \\
\text { Chemical release }\end{array}$} & \multirow[b]{2}{*}{2} & \multirow[b]{2}{*}{$\begin{array}{l}\text { Extremely } \\
\text { unlikely }\end{array}$} & \multirow[b]{2}{*}{ Hazardous chemical release } \\
\hline & $\begin{array}{l}\text { unlikely } \\
\text { (see event tree G) }\end{array}$ & & & & \\
\hline & Leak or & $\begin{array}{l}\text { unlikely } \\
\text { Hood ventilation } \\
\text { System mitigates } \\
\text { Chemical release } \\
\end{array}$ & 3 & & \\
\hline & $\begin{array}{l}\text { Spill } \\
\text { Of container }\end{array}$ & \multirow{2}{*}{$\begin{array}{l}\text { Hood ventilation } \\
\text { System fails to mitigate } \\
\text { Chemical release }\end{array}$} & \multirow[b]{2}{*}{4} & \multirow[b]{2}{*}{\begin{tabular}{|l}
$\begin{array}{l}\text { Extremely } \\
\text { unlikely }\end{array}$ \\
\end{tabular}} & \multirow[b]{2}{*}{ Hazardous chemical release } \\
\hline & unlikely & & & & \\
\hline & & unlikely & & & \\
\hline \multirow[t]{7}{*}{ likely } & $\begin{array}{l}\text { Fire } \\
\text { In }\end{array}$ & $\begin{array}{l}\text { Hood ventilation } \\
\text { System mitigates } \\
\text { Chemical release } \\
\end{array}$ & 5 & & \\
\hline & $\begin{array}{l}\text { Proximity } \\
\text { Of chemicals }\end{array}$ & \multirow{2}{*}{$\begin{array}{l}\text { Hood ventilation } \\
\text { System fails to mitigate } \\
\text { Chemical release } \\
\text { unlikely }\end{array}$} & \multirow[b]{2}{*}{6} & \multirow[b]{2}{*}{$\begin{array}{l}\text { Extremely } \\
\text { unlikely }\end{array}$} & \multirow[b]{2}{*}{ Hazardous chemical release } \\
\hline & $\begin{array}{l}\text { unlikely } \\
\text { (see scenario F) }\end{array}$ & & & & \\
\hline & $\begin{array}{l}\text { chemicals } \\
\text { In proximity } \\
\text { To accidental }\end{array}$ & $\begin{array}{l}\text { unlikely } \\
\text { Hood ventilation } \\
\text { System mitigates } \\
\text { Chemical release } \\
\end{array}$ & 7 & & \\
\hline & $\begin{array}{l}\text { Explosives } \\
\text { Initiation }\end{array}$ & \multirow[b]{2}{*}{$\begin{array}{l}\text { Hood ventilation } \\
\text { System fails to mitigate } \\
\text { Chemical release }\end{array}$} & \multirow{3}{*}{\multicolumn{2}{|c|}{$\begin{array}{l}\text { Extremely } \\
\text { unlikely }\end{array}$}} & \multirow{3}{*}{ Hazardous chemical release } \\
\hline & $\begin{array}{l}\text { Extremely unlikely } \\
\text { (see scenario A) }\end{array}$ & & & & \\
\hline & & likely & & & \\
\hline
\end{tabular}


Fig N-- SAR Scenario N. Radiological dispersion/release: Radiological materials are dispersed due to combination of spill and fire.

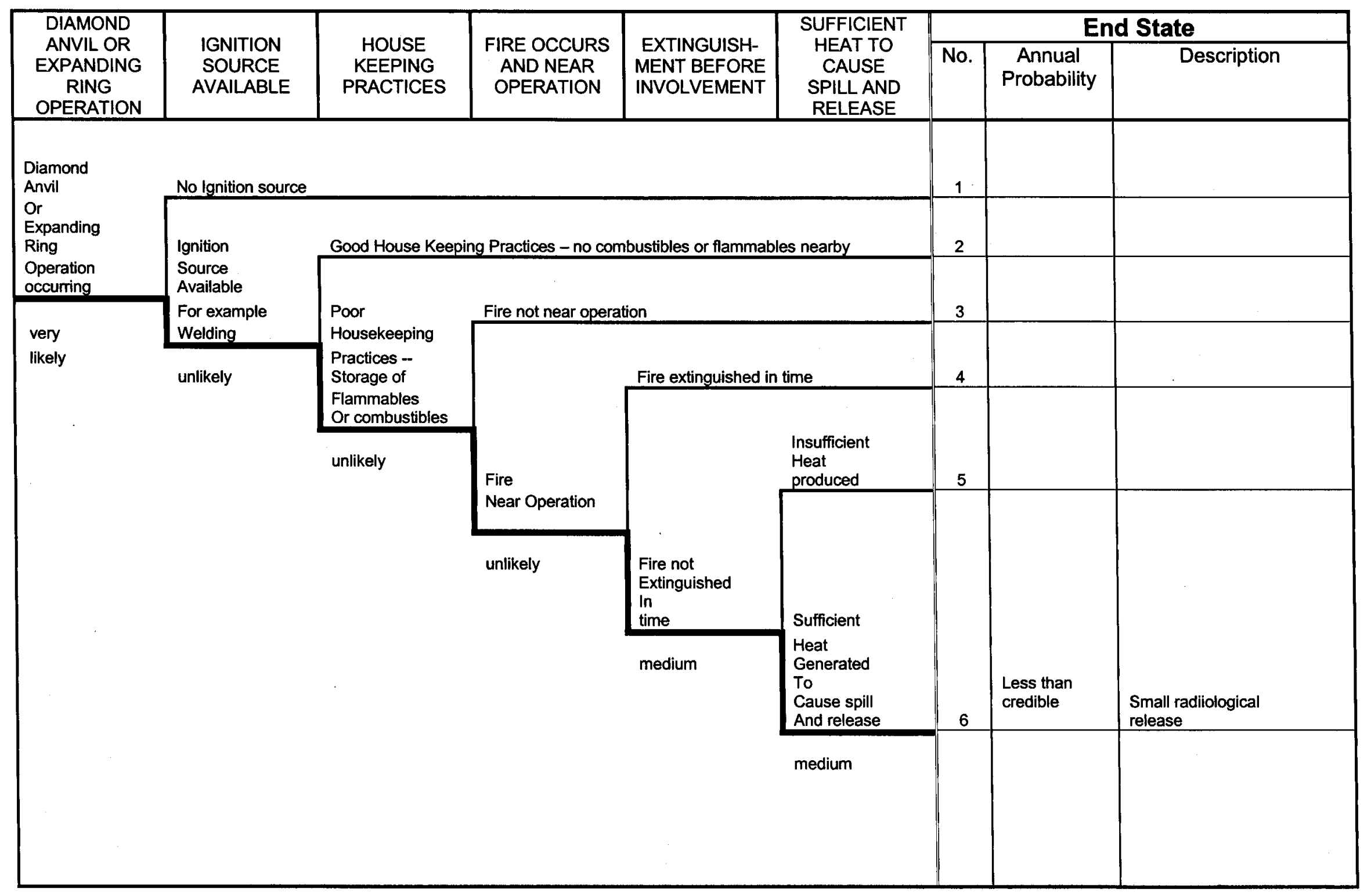


Fig O - SAR Scenario O -- Chemical exposure: Personnel are exposed to toxic or asphyxiant gases or radiological contamination from re-entry into firing tanks after an experiment

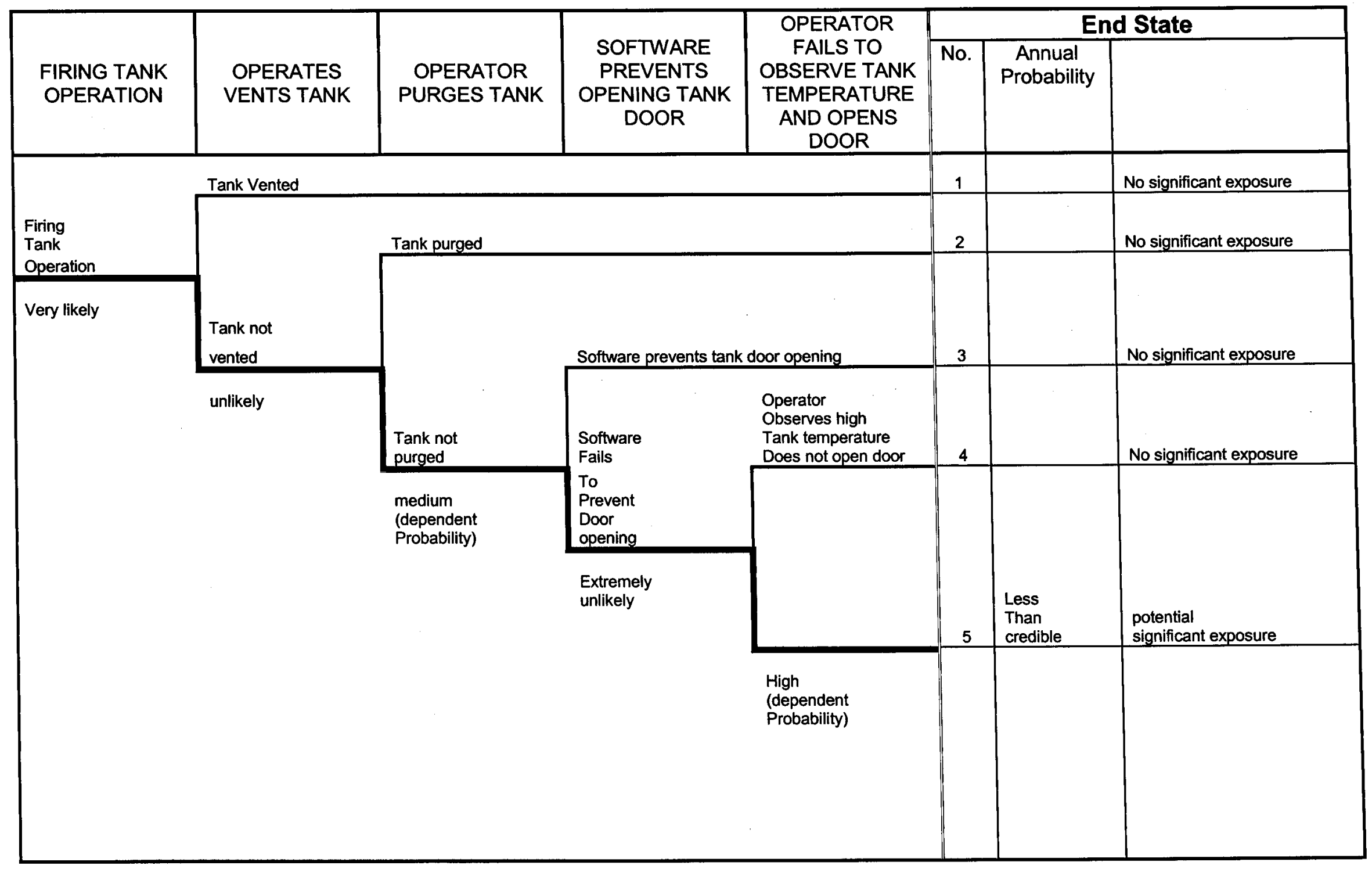


Fig P -- SAR Scenario P - Ionizing radiation exposure: Personnel are exposed to radiation from radiation generating devices (e.g. x-ray heads)

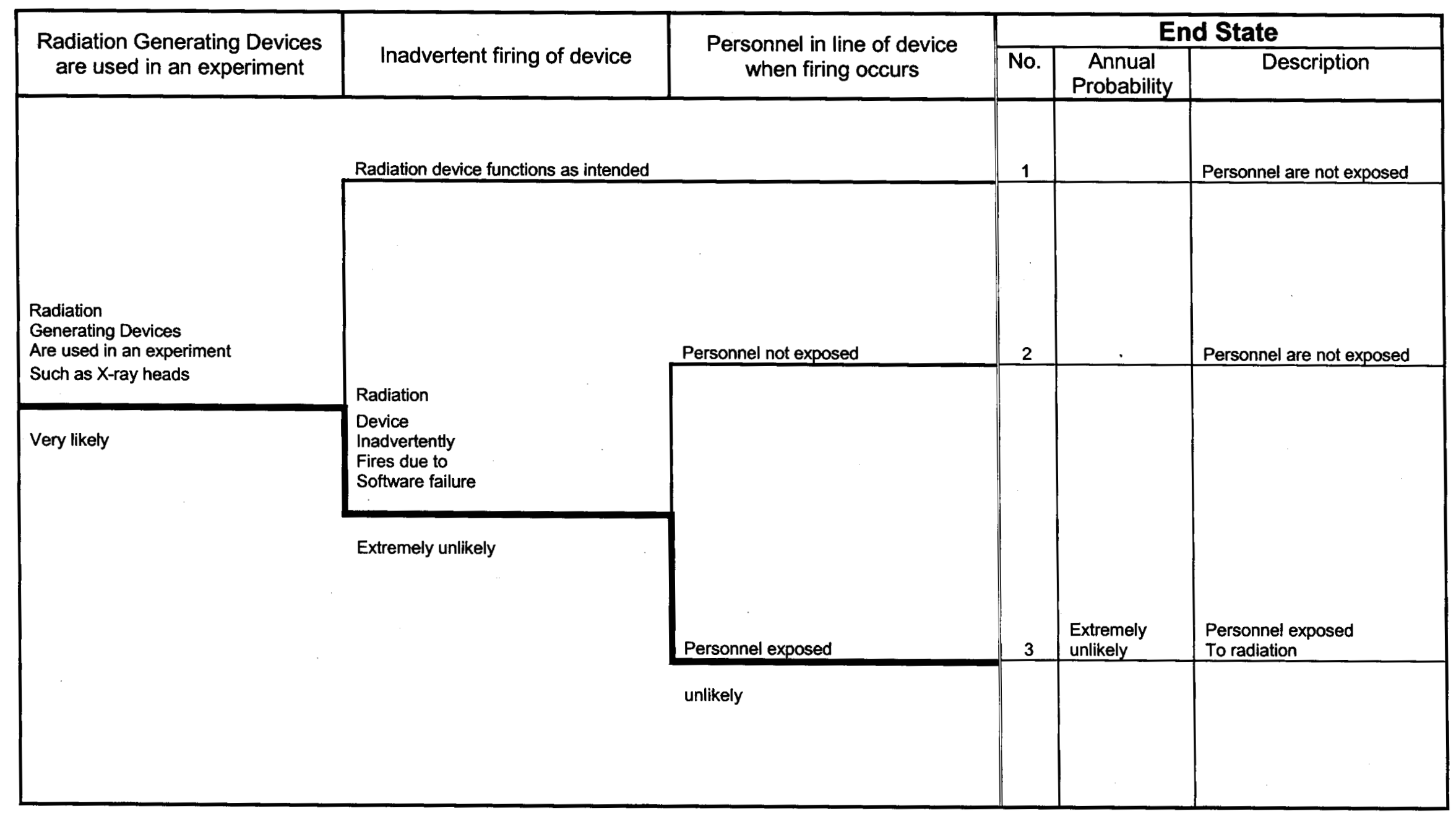


Fig Q - SAR Scenario Q -- Non-ionizing radiation exposure: Personnel are injured from exposure to laser light

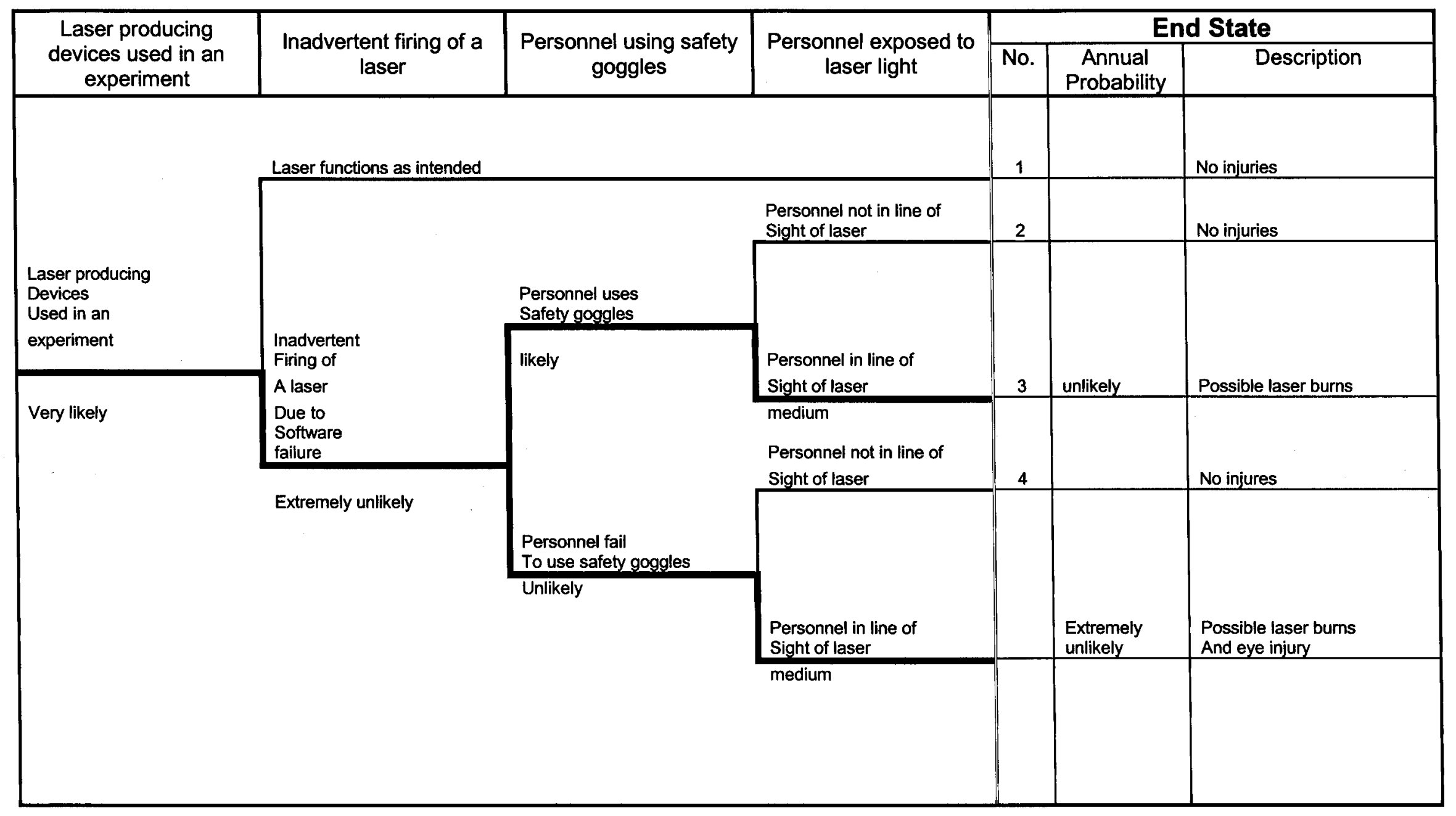


Fig. S - Scenario S -- Personnel exposed to overpressure: Secondary burnoff in firing tank ruptures exhaust ventilation ducting.

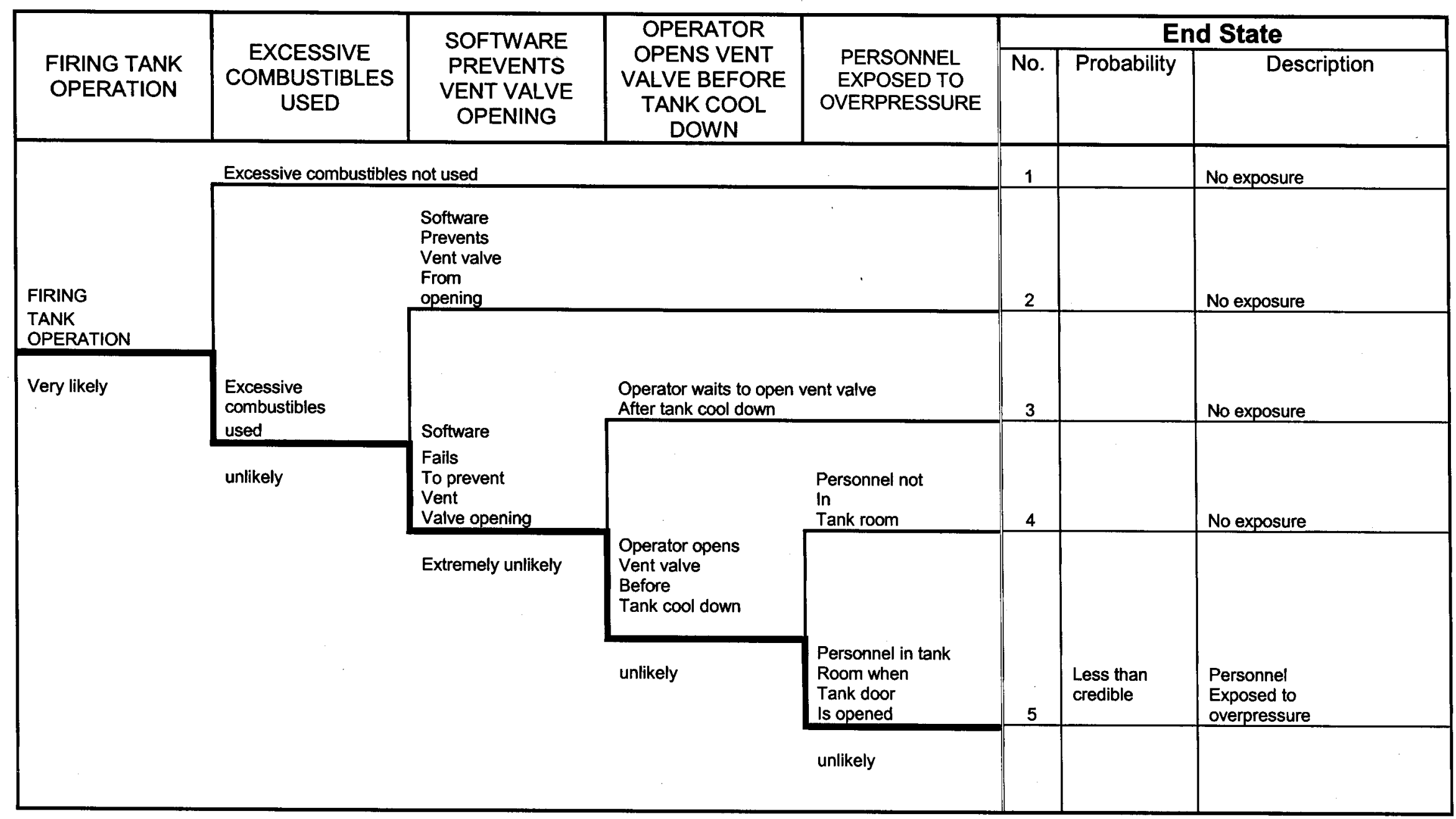




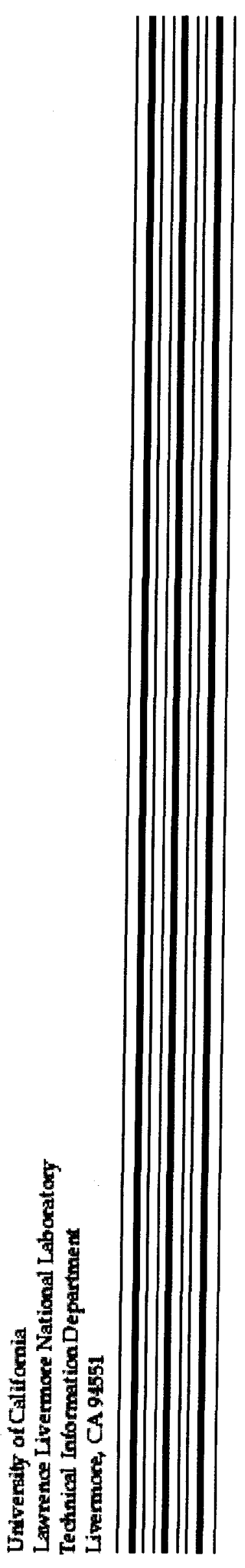

